\title{
Sous-catégories réflexives et la théorie générale des radicaux *
}

par

\author{
François Carreau (Montréal)
}

La notion de radical utilisée en théorie des anneaux, puis en celles des modules sur un anneau et des groupes, a fait l'objet de plusieurs théories. Elles se ramènent principalement à trois: celle des propriétës radicales de Šul'geifer [13] pour une catégorie dans laquelle sont valides les théorèmes classiques d'isomorphisme; puis celle de radical sous-foncteur de l'identité de Maranda [9] et de Dickson [4] pour une catégorie abélienne; enfin celle d'Amitsur [1], les RI-propriétés, et la définition d'un radical d'anneau selon Michler [10]. Cet article a pour but de présenter une théorie encore plus générale pour obtenir de nouveaux résultats concernant le radical et comparer ces différentes théories entre elles.

La première section comporte l'étude d'une généralisation de la notion de sous-catégorie réflexive: les sous-catégories $\boldsymbol{M}$-réflexives d'une catégorie $\boldsymbol{C}, \boldsymbol{M}$ étant une sous-catégorie de $\boldsymbol{C}$ possédant la même classe d'objets que $\boldsymbol{C}$. Cette notion est en quelque sorte le correspondant dual de celle de radical. Dans le cas d'une catégorie $\boldsymbol{C}$ dans laquelle tout morphisme possède une image au sens de Kennison [6], nous construisons la plus petite sous-catégorie $\boldsymbol{M}$-réflexive de $\boldsymbol{C}$ contenant une sous-catégorie donnée, puis la plus petite sous-catégorie réflexive contenant cette même sous-catégorie. Et enfin, nous donnons une construction qui implique un foncteur ayant un adjoint à gauche.

La deuxième section concerne les catégories dans lesquelles tout morphisme se décompose en conoyau de son noyau et en monomorphisme. Nous étudions alors les sous-catégories $N$-réflexives d'une telle catégorie $\boldsymbol{C}$, les morphismes de $\boldsymbol{N}$ étant les conoyaux de $\boldsymbol{C}$. Si on prend comme radical d'un objet le noyau de sa $\boldsymbol{N}$-réflexion, il est montré que l'on

* Cet article contient pour une partie des résultats d'une thèse de doctorat écrite ous la direction du Professeur Jean Maranda, que l'auteur remercie pour l'aide et les conseils qu'il lui a donnés. 
retrouve la définition de Michler pour un radical d'un anneau. En appliquant une des constructions de la section précédente, on obtient un radical à partir d'une classe quelconque d'objets. Nous précisons ensuite les conditions pour qu'il y ait une dualité complète entre la classe d'objets semi-simples et celle des objets radicaux pour un radical donné, dualité déjà notée par Rjabuhin [12] entre autres. Et enfin nous pouvons comparer les autres définitions de la notion de radical avec la précédente.

La troisième section traite des radicaux dans la catégorie des anneaux avec élément-unité et des homomorphismes qui appliquent l'unité sur l'unité: on ne peut y définir un radical en terme d'idéaux, puisque ceux-ci n'existent pas dans cette catégorie. On peut très bien cependant considérer les sous-catégories $\boldsymbol{S}$-réflexives, les morphismes de $\boldsymbol{S}$ étant les surjections. Par une application d'un résultat de la première section, nous montrons qu'il suffit, dans le cas des radicaux utilisés dans la théorie des anneaux, de les définir pour les anneaux avec élément-unité, ainsi que le fait Bourbaki [3] pour le radical de Jacobson. Dans la dernière partie, on résoud le problème suivant: pour chaque anneau $A$, considérant un radical dans la catégorie des $A$-modules à droite unitaires, on se demande quelles sont les conditions pour que le radical de $A$ considérée comme un $A$-module devienne un radical dans la catégorie des anneaux. Les conditions trouvées ne concernent qu'une classe particulière de $A$-modules, c'est-à-dire une classe d'idéaux à droite de l'anneau.

\section{Les sous-catégories $M$-réflexives}

Pour les notions usuelles qui se rapportent aux catégories, nous renvoyons le lecteur au livre de Mitchell [11], en notant cependant que nous utiliserons les termes "réflexif", "réflecteur" et „réflexion" au lieu de „coréflexif", etc.... et réciproquement.

§1. Définition. Une sous-catégorie $\boldsymbol{M}$ d'une catégorie $\boldsymbol{C}$ est dite coextensive si la classe des objets de $\boldsymbol{M}$ est égale à celle de $\boldsymbol{C}$, i.e. si Ob $\boldsymbol{M}$ $=\mathrm{Ob} C$. Nous notons $\boldsymbol{B} \stackrel{\stackrel{R}{\mathrm{C}}}{\mathrm{C}}$

de la catégorie $\boldsymbol{C}$ avec $R$ : $\boldsymbol{C}$ si $\boldsymbol{B}$ est une sous-catégorie pleine réflexive la. réflexion de $\boldsymbol{C}$ dans $\boldsymbol{B} \rightarrow \boldsymbol{B}$ comme foncteur-réflecteur et $\varrho: 1_{\boldsymbol{C}} \rightarrow R$ Soit $\boldsymbol{M}$ une catégorie

gorie $\boldsymbol{B}$ de $\boldsymbol{C}$ est appelée sous-catégorie ${ }^{-}$d'une catégorie $\boldsymbol{C}$. Une sous-catéà dire que tout objet $A$ de $\boldsymbol{C}$ possède $\boldsymbol{M}$-réflexive si $\boldsymbol{B} \bar{C} \boldsymbol{M}$ : ce qui revient àr une propriété universelle restreine "réflexion" dans $\boldsymbol{B}$, caractérisée Une prostreinte aux morphismes de $\boldsymbol{M}$. réflexive. Il est facile de vérifier, à titre d'exemples, que la sous-catégorie pleine de tous les anneaux semi-simples pour un radical au sens de Divinsky [5] est sous-catégorie $\boldsymbol{M}$-réflexive de la catégorie de tous les anneaux, $\boldsymbol{M}$ ne contenant que les homomorphismes surjectifs. Dans la seconde section, nous considérons une eatégorie $\boldsymbol{C}$ dans laquelle le produit de deux épimorphismes normaux est un épimorphisme normal au sens de ([8], § 8); si $\boldsymbol{N}$ est la sous-catégorie coextensive de $\boldsymbol{C}$ constituée de tous les épimorphismes normaux, alors les sous-catégories $\boldsymbol{N}$-réflexites de $\boldsymbol{C}$ détermineront les radicaux dans $\boldsymbol{C}$.

Nous allons construire des sous-catégories $\boldsymbol{M}$-réflexives particulières d'une catégorie dont tout morphisme possède une image, c'est-à-dire une $(\mathcal{J}, J)$-décomposition.

§ 2. Catégories avec $(\mathfrak{T}, \mathfrak{J})$-décomposition. Nous reprenons la théorie présentée par Kennison [6]. Soient une catégorie $\boldsymbol{C}$ et deux classes $\mathfrak{I}$ et $\mathfrak{J}$ de morphismes de $C$. $C$ est catégorie avec $(\mathfrak{T}, \mathfrak{J})$-décomposition (right bicategory structure) si sont vérifiés les axiomes suivants:

A1. tout isomorphisme de $\boldsymbol{C}$ appartient à $\mathfrak{T} \cap J$,

A2. $\mathcal{J}$ et $\mathfrak{J}$ sont respectivement fermées sous la composition de morphismes,

A3. tout morphisme $f$ de $C$ se décompose ainsi: $f=h g$ pour $g \in \mathfrak{T}$ et $h \in \mathcal{J}$. De plus si $f=h^{\prime} g^{\prime}$ avec $g^{\prime} \in \mathcal{I}$ et $h^{\prime} \in \mathfrak{J}$, alors il existe un isomorphisme $e$ tel que $e g=g^{\prime}$ et $h^{\prime} e=h$,

A4. tout morphisme de $\mathfrak{T}$ est un épimorphisme.

Propostrion 1.1. Soit $\boldsymbol{C}$ une catégorie avec $(\mathfrak{T}, \mathfrak{J})$-décomposition. Alors

(1) $\mathfrak{I} \cap \mathfrak{J}=$ la classe de tous les isomorphismes de $\boldsymbol{C}$,

(2) $g f \in \mathfrak{J}$ implique que $f \in \mathfrak{J}$,

(3) $g f \in \mathfrak{T}$ et $f$ épimorphisme impliquent que $g \in \mathfrak{T}$.

(4) Pour tout diagramme commutatif de la forme suivante:

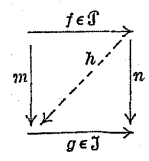

il existe $h \in C$, rendant tout commutatif.

§ 3. Sous-catégories $\boldsymbol{P}$-réflexives d'une catégorie avec $(\mathfrak{T}, \mathfrak{J})$-décomposition. Soit $\boldsymbol{C}$ une catégorie avec $(\mathfrak{T}, \mathfrak{J})$ - décomposition. Si $\boldsymbol{P}$ est la sous-catégorie coextensive de $\boldsymbol{C}$ dont les morphismes sont ceux de $\mathfrak{T}$, on peut alors considérer les sous-catégories $\boldsymbol{P}$-réflexives de $\boldsymbol{C}$. Dans la eatégorie $\boldsymbol{C}$, on peut définir les notions suivantes: 
Définition 1. Soient $A \in \mathrm{Ob} \boldsymbol{C}$ et $u \in \mathfrak{J} ;(A, u)$ est un $(\mathfrak{J}, \mathfrak{J})$-produit d'une famille $\left\{B_{i}\right\}_{i \in I}$ d'objets de $\boldsymbol{C}$ si le produit $\prod_{i \in I} B_{i}$ existe dans $\boldsymbol{C}$ et si $u: A \rightarrow \prod_{i \in I} B_{i}$ est tel que $p_{i} u \in \mathcal{T}$ pour tout $p_{i}$, la projection de $\prod_{i \in I} B_{i}$ sur la $i^{e}$ composante $B_{i}$.

Déflnition 2. Une classe $\mathfrak{B}$ d'objets de $\boldsymbol{C}$ est une base d'une souscatégorie $\boldsymbol{A} \boldsymbol{P}$-réflexive de $\boldsymbol{C}$ si pour tout $A \in \mathrm{Ob} \boldsymbol{A}$, il existe $u \in \mathcal{J}$ tel que $(\mathcal{A}, u)$ est un $(\mathfrak{S}, \mathfrak{J})$-produit d'objets de $\mathfrak{B}$.

DÉFLnITION 3. Une catégorie $\boldsymbol{C}$ est $\boldsymbol{P}$-, „cowell-powered" pour une classe $\mathcal{B}$ d'objets de $\boldsymbol{C}$ si pour tout $A \in \mathrm{Ob} \boldsymbol{C}$, la classe $\{f \in \mathcal{T} \mid f: A \rightarrow B$ avec $B \in \Re\}$ modulo la relation $\sim$ est un ensemble, la relation $\sim$ étant ainsi définie: $f \sim g$ s'il existe un isomorphisme $e$ tel que $e f=g$.

On rappelle qu'une sous-catégorie $\boldsymbol{B}$ de $C$ est replète si tout $A \in \mathrm{Ob} \boldsymbol{C}$ tel qu'il existe un isomorphisme $e: A \rightarrow B$ arec $B \in \boldsymbol{B}$ est dans $\mathrm{Ob} \boldsymbol{B}$.

La proposition suivante montre qu'une sous-catégorie $\boldsymbol{P}$-réflexive replète de $\boldsymbol{C}$ est fermée sous la formation de $(\mathfrak{T}, \mathfrak{J})$-produits.

Proposition 1.2. Soient $\boldsymbol{C}$ une catégorie avec $(\mathfrak{T}, \mathfrak{J})$-décomposition et $\boldsymbol{B}$ une sous-catégorie replète $\boldsymbol{P}$-réflexive de $\boldsymbol{C}$. Si $(A, u)$ est un $(\mathfrak{S}, \mathfrak{J})$-produit de $\left\{B_{i}\right\}_{i \in I}, B_{i} \in \mathrm{Ob} \boldsymbol{B}$, alors $A$ est dans $\mathrm{Ob} \boldsymbol{B}$.

Démonstration. Pour chaque $i \in I, p_{i} u: A \rightarrow \prod_{i \in I} B_{i} \rightarrow B_{i} \in \mathcal{I}$ et $B_{i} \in \mathrm{ObB}:$ d'où il existe $g_{i}: R(A) \rightarrow B_{i}$ tel que $g_{i \varrho_{A}}=p_{i} u(\boldsymbol{B} \stackrel{R}{\stackrel{R}{\leftrightarrows}} \boldsymbol{P})$. Ainsi il existe $h: R(A) \rightarrow \prod_{i \in I} . B_{i}$ tel que $p_{i} h=g_{i}$. Comme $p_{i} u=g_{i} \varrho_{A}=p_{i} h_{\varrho_{A}}$, alors $u=h_{\varrho_{A}} \epsilon$ J: d'après la proposition 1.1, $\varrho_{A}$ est un isomorphisme. Done $A \in \mathrm{Ob} B$.

$\mathrm{Si} \mathfrak{B}$ est une classe d'objets de $\boldsymbol{C}$, soit la sous-catégorie $\boldsymbol{B}$ pleine de $\boldsymbol{P}$ dont $\mathrm{Ob} \boldsymbol{B}=\Re$. Nous pouvons alors construire la plus petite souscatégorie replète $\boldsymbol{P}$-réflexive de $\boldsymbol{C}$ qui contienne $\boldsymbol{B}$.

THÉOR亡̀ME 1.3. Soient une catégorie $C$ avec $(\mathfrak{T}, \mathfrak{J})$-décomposition et une sous-catégorie $\boldsymbol{B}$ de $\boldsymbol{P}$. A la condition que $\boldsymbol{C}$ possède des produits quelconques et soit $\mathfrak{T}$-,,cowell-powered" pour $\mathrm{Ob} \boldsymbol{B}$, il existe une sous-catégorie $\boldsymbol{A}$ replète $\boldsymbol{P}$-réflexive, la plus petite contenant $\boldsymbol{B}$.

Démonstration. Soit $\boldsymbol{A}$ la sous-catégorie pleine de $\boldsymbol{P}$ formée de tous les $(\mathfrak{T}, \mathfrak{J})$-produits de $\mathrm{Ob} \boldsymbol{B}$. Pour tout $A \in \mathrm{Ob} \boldsymbol{C}$, soit une $(\mathfrak{T}, \mathfrak{J})$-décomposition du morphisme $f: A \rightarrow \prod_{I} B_{i}$, tel que $p_{i} f=f_{i}: A \rightarrow B_{i} \in \mathcal{T}$ avec $B_{i} \in \mathrm{Ob} \boldsymbol{B}, I$ étant l'ensemble des classes d'équivalence $\{a \in \mathcal{I} \mid a: A \rightarrow B$ arec $B \in \mathrm{Ob} \boldsymbol{B}\}: f=h_{\varrho_{A}}$. Posons $\varrho_{A}: A \rightarrow R(A)$. Alors pour tout $i \in I$, comme $f_{i}$ et $\varrho_{A}$ sont dans $\mathfrak{T}, f_{i}=p_{i} f=\left(p_{i} h\right) \varrho_{A}$ implique $p_{i} h \in \mathcal{J}$. Comme $h \in \mathcal{J}$, alors $R(A) \in \mathrm{Ob} \boldsymbol{A}$. Soit maintenant $k: A \rightarrow C \in \mathcal{I}$ tel que $C \in \mathrm{Ob} \boldsymbol{A}$, i.e. il existe $\left\{D_{j}\right\}_{j \in J} \subseteq \mathrm{Ob} \boldsymbol{B}$ et $v: C \rightarrow \prod_{J} D_{j} \in \mathcal{J}$ tel que $q_{j} v: C \rightarrow \prod_{J} D_{j} \rightarrow D_{j}$ est dans $\mathfrak{S}$ pour tout $j \in J$. Alors on complète le diagramme suivant:

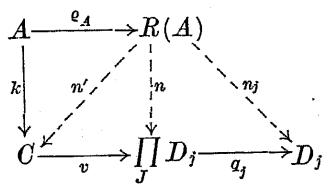

comme $\left(q_{j} v\right) z_{i} \in \mathcal{T}$ et $D_{j} \in \mathrm{Ob} \boldsymbol{B}$, il existe $i_{j} \in I$ tel que $p_{i j} f \sim q_{j} v k$, i.e. tel 'lil existe un isomorphisme $e_{j}$ et $e_{j} p_{i_{j}} f=q_{j} v k$. Posons $n_{j}=e_{j} p_{i_{j}} h$; comme pour tout $j \in J, n_{j} \varrho_{A}=e_{j} p_{i j} h_{\varrho_{A}}=e_{j} p_{i j} f=q_{j} v k$, il existe $n: R(A)$ $\rightarrow \prod D_{j}$ tel que $n \varrho_{A}=v k$. Puisque $\varrho_{A} \in \mathcal{T}$ et $v \in \mathcal{J}$, il existe $n^{\prime}: R(A) \rightarrow C$ (proposition 1.1) tel que $k=n^{\prime} \varrho_{A}$; et $n^{\prime}$ est unique puisque $\varrho_{A}$ est un épimorphisme. Donc $\varrho_{A}$ est la réflexion de $A$ dans $\boldsymbol{A}$, et $\boldsymbol{A} \stackrel{R}{\complement} \boldsymbol{P}$

La proposition 1.3 implique que $\boldsymbol{A}$ soit la plus petite sous-catégorie eplète $\boldsymbol{P}$-réflexive contenant $\boldsymbol{B}$.

COROLLAIre 1.4. Si $\Re \subset \mathrm{Ob} C$ est une base d'une sous-catégorie $\boldsymbol{A}$ replète $\boldsymbol{P}$-réflexive de $\boldsymbol{C}$, alors $\boldsymbol{A}$ est la plus petite sous-catégorie $\boldsymbol{P}$-réflexive contenant la sous-catégorie $\boldsymbol{B}$ pleine de $\boldsymbol{P}$ tel que $\mathrm{Ob} \boldsymbol{B}=\Re_{3}$.

Il est possible d'utiliser la construction du théorème 1.3 pour obtenir la plus petite sous-catégorie réflexive contenant une sous-catégorie donnée, moyennant la notion suivante:

Dḱfinitron 4 . Une base $\mathcal{B}$ est dite J-héréditaire si pour tout $u: A \rightarrow$ $\rightarrow B \in \mathcal{J}$ et $B \in \mathcal{B}$, alors $A \in \mathcal{B}$.

Proposition 1.5. Soit $\mathfrak{B}$ une base d'une sous-catégorie $\boldsymbol{A}$ replète $\boldsymbol{P}$-réflexive d'une catégorie $\boldsymbol{C}$ avec $(\mathfrak{S}, \mathfrak{J})$-décomposition. Si $\mathfrak{B}$ est J-héréditaire, alors $\boldsymbol{A} \stackrel{\mathbb{R}}{\complement} \boldsymbol{C}$ avec des réflexions dans $\mathfrak{T}$.

Démonstration. Soit $f: A \rightarrow B \in C$ tel que $B \in \mathrm{Ob} A$. Prenons une $(\mathfrak{T}, \mathfrak{J})$-décomposition de $f: f=v f^{\prime}: A \rightarrow C \rightarrow B$. Il suffit de vérifier que $C \in \operatorname{Ob} \boldsymbol{A}$. En effet il existe $\left\{B_{i}\right\}_{i \in I} \subseteq \mathcal{B}$ et $u: B \rightarrow \prod_{I} B_{i} \in \mathcal{J}$ tel que $p_{i} u \in \mathcal{J}$ pour tout $i \in I$. Soit $n_{i} m_{i}$ une $(\mathfrak{T}, \mathfrak{J})$-décomposition de $p_{i} u v ; m_{i}: C \rightarrow D_{i}$ et $n_{i}: D_{i} \rightarrow B_{i}$. Alors il existe $n: \prod_{I} D_{i} \rightarrow \prod_{I} B_{i}$ tel que $p_{i} n=n_{i} q_{i}$ et aussi $k: C \rightarrow \prod_{I} D_{i}$ tel que $q_{i} k=m_{i}$. Le diagramme

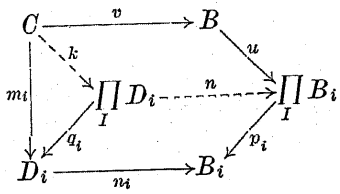


est entièrement commutatif. En effet $p_{i} u v=n_{i} m_{i}=n_{i} q_{i} k_{i}=p_{i} n k$ pour tout $i \in I$; d'où $u v=n k \in \mathcal{J}$. Or $D_{i} \in \mathfrak{B}$ puisque $\mathcal{B}$ est $J$-héréditaire et $k \in \mathfrak{J}$

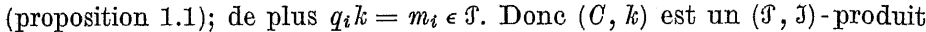
d'objets de $\mathcal{B}$, i.e. $C \in \mathrm{Ob} A$, (proposition 1.2).

Propostmon 1.6. Soit $\boldsymbol{B}$ une sous-catégorie replète réflexive d'une

catégorie $\boldsymbol{C}$ avec $(\mathfrak{S}, \jmath)$ )-décomposition; si $\boldsymbol{B} \stackrel{R}{\leftarrow} \boldsymbol{C}$ avec des réflexions dans $\mathfrak{S}$, alors $u$ : $A \rightarrow B \in \mathfrak{J}$ tel que $B \in \mathrm{ObB}$ implique $A \in \mathrm{Ob} \boldsymbol{B}$.

Démonstration. Soit $u: A \rightarrow B \in \mathfrak{J}$ avec $B \in \mathrm{ObB}$. Puisque $\boldsymbol{B} \stackrel{R}{\complement} C$, $\varrho_{B} u=R(u) \varrho_{A}$ et, $\varrho_{B}$ étant un isomorphisme, alors $\varrho_{A}$ l'est aussi (proposition 1.1). Done $A \in \mathrm{ObB}$.

La proposition précédente signifie qu'une sous-catégorie replète réflexive avec réflexions dans $\mathfrak{T}$ est „fermée sous les $J$-sous-objets". Des deux propositions précédentes, nous obtenons:

THÉORÈME 1.7. Soient une catégorie $\boldsymbol{C}$ avec $(\mathfrak{T}, \mathfrak{J})$-décomposition et $\boldsymbol{B}$ une sous-catégorie de $\boldsymbol{P}$. Alors il existe une plus petite sous-catégorie $\boldsymbol{A}$ replète réflexive de $\boldsymbol{C}$ avec des réflexions dans $\mathfrak{T}$ contenant $\boldsymbol{B}, \grave{a}$ la condition

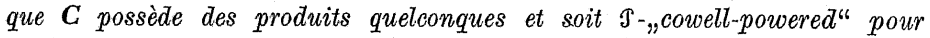
$\mathcal{B}^{\prime}=\{A \in \mathrm{Ob} \boldsymbol{C} \mid u: A \rightarrow B \in \mathfrak{J}$ avec $B \in \mathrm{Ob} \boldsymbol{B}\}$

Il suffit d'appliquer le théorème 1.3. à la sous-catégorie pleine $\boldsymbol{B}^{\prime}$ de $\boldsymbol{P}$ tel que $\mathrm{Ob} \boldsymbol{B}^{\prime}=\mathfrak{B}^{\prime}$.

Nous allons maintenant çonsidérer un foncteur $T: \boldsymbol{D} \rightarrow \boldsymbol{C}$ d'une catégorie $\boldsymbol{D}$ quelconque dans une catégorie $\boldsymbol{C}$ avec $(\mathfrak{J}, \mathfrak{J})$-décomposition en supposant que $S: C \rightarrow \boldsymbol{D}$ est l'adjoint à gauche de $T$. Soit $\boldsymbol{M}$ une souscatégorie coextensive de $\boldsymbol{D}$. Si $\boldsymbol{B}$ est une sous-catégorie $\boldsymbol{M}$-réflexive de $\boldsymbol{D}$, on peut se demander si $\boldsymbol{B}$ n'induit pas une sous-catégorie $\boldsymbol{P}$-réflexive de $C$, en composant en quelque sorte les adjoints. Nous notons $x: 1_{C} \rightarrow T S$, le morphisme-adjonction.

THÉorìme 1.8. Soit $T: \boldsymbol{D} \rightarrow C$ un foncteur d'une catégorie $\boldsymbol{D}$ quelconque dans une catégorie $\boldsymbol{C}$ avec $(\mathfrak{T}, \mathfrak{J})$-décomposition tel que $S: C \rightarrow \boldsymbol{D}$ est son adjoint à gauche. Soit $\boldsymbol{B}$ une sous-catégorie $\boldsymbol{M}$-réflexive de $\boldsymbol{D}$. Si $\boldsymbol{M}$ possède la propriété que $g f \in \boldsymbol{M}$ et $f \in \boldsymbol{M}$ implique $g \in \boldsymbol{M}$ et si $S(f)$ est dans $\boldsymbol{M}$ pour tout $f \in \mathfrak{T}$, alors la sous-catégorie $\boldsymbol{A}$ pleine de $\boldsymbol{P}$ dont la classe des objets est

$$
\left\{A \in \mathrm{Ob} C \mid A \stackrel{*_{A}}{\longrightarrow} T S(A) \stackrel{T\left(Q_{S(A)}\right)}{\longrightarrow} T R S(A) \in I\right\}
$$

$\varrho: 1_{M} \rightarrow R$ étant la réflexion de $\boldsymbol{M}$ dans $\boldsymbol{B}$, est une sous-catégorie $\boldsymbol{P}$-réflesive de $\boldsymbol{C}$.

Démonstration. Pour tout $A \in \mathrm{ObC}$, posons $u \bar{\varrho}_{A}: A \rightarrow \bar{R}(A) \rightarrow$ $\rightarrow T R S(A)$ une $\left(\mathfrak{S}\right.$, J)-décomposition de $T\left(\varrho_{S(A)}\right) x_{A}: A \rightarrow T S(A) \rightarrow T R S(A)$.
(1) $\bar{R}(A) \in \mathrm{Ob} A$. Comme $S$ est l'adjoint à gauche de $T$, il existe $g: S \bar{R}(A) \rightarrow R S(A)$ tel que $u=T(g) x_{\bar{R}(A)}$. Alors $T(g) T S\left(\bar{\varrho}_{A}\right) x_{A}=T(g) x_{\bar{R}(A)} \bar{\varrho}_{A}$ $=u \bar{\varrho}_{A}=T\left(\varrho_{S(A)}\right) \varkappa_{A}$; d'où $g S\left(\bar{\varrho}_{A}\right)=\varrho_{S(A)}$. Par conséquent le diagramme

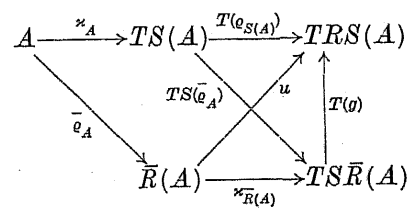

est commutatif. Comme $\varrho_{S(A)}$ et $S\left(\bar{\varrho}_{A}\right)$ sont dans $\boldsymbol{M}$, alors $g \in \boldsymbol{M}$. Soit $h: S \bar{R}(A) \rightarrow B \in \boldsymbol{M}$ tel que $B \in \mathrm{Ob} \boldsymbol{B}$. Comme $h S\left(\bar{\varrho}_{A}\right) \in \boldsymbol{M}$, il existe $h^{\prime}$ : $R S(A) \rightarrow B \in \boldsymbol{M}$ tel que $h^{\prime} \varrho_{S(A)}=h S\left(\bar{\varrho}_{A}\right)$. D'où $h^{\prime} g S\left(\bar{\varrho}_{A}\right)=h S\left(\bar{\varrho}_{A}\right)$ et $h^{\prime} g=h, S$ préservant les épimorphismes. $h^{\prime}$ est évidemment unique; par conséquent il existe un isomorphisme $e: R S(A) \rightarrow R S \bar{R}(A)$. Donc $T\left(\varrho_{S \bar{R}(A)}\right) x_{\bar{R}(A)} \in \mathcal{J}$, comme $u \in \mathcal{J}$.

(2) Soient $g: A \rightarrow B \in \mathfrak{I}$ tel que $B \in \mathrm{Ob} \boldsymbol{A}$ et $u \bar{\varrho}_{A}: A \rightarrow \bar{R}(A) \rightarrow T R S(A)$ une $(\mathcal{T}, \mathfrak{J})$-décomposition de $T\left(\varrho_{S(A)}\right) \varkappa_{A}: A \rightarrow T S(A) \rightarrow T R S(A)$; alors le diagramme

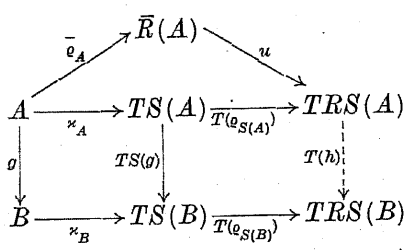

est commutatif. Comme $S(g) \in \boldsymbol{M}$ et $\varrho_{S(B)} \in M$, il existe $h: R S(A) \rightarrow R S(B)$ tel que $h_{S(A)}=\varrho_{S(B)} S(g)$. Par suite $(T(h) u) \bar{\varrho}_{A}=\left(T\left(\varrho_{S(B)}\right) r_{B}\right) g$ tel que $\bar{\varrho}_{A} \in \mathfrak{S}$ et $T\left(\varrho_{S(B)}\right) \varkappa_{B} \in \mathfrak{J}$ : done (proposition 1.1) il existe $h^{\prime}: \bar{R}(A) \rightarrow B$ tel que $g=h^{\prime} \varrho_{A}$ et $h^{\prime}$ est unique avec cette propriété puisque $\bar{\varrho}_{A}$ est un épimorphisme. Donc $\boldsymbol{A} \stackrel{\overline{\mathrm{C}}}{\bar{C}} \boldsymbol{P}$ et $\bar{\varrho}: 1_{\boldsymbol{P}} \rightarrow \bar{R}$ est la réflexion de $\boldsymbol{P}$ dans $\boldsymbol{A}$.

Corollatre 1.9. Si $\boldsymbol{M}=\boldsymbol{D}$, alors $\boldsymbol{A}$ est une sous-catégorie réflexive de $\boldsymbol{C}$ aveo des réflexions dans $\mathfrak{S}$.

En effet les conditions du théorème 1.8 sont réalisées et dans (2) de la démonstration, on peut prendre $g \in \boldsymbol{C}$.

\section{Théorie générale des radicaux}

Nous allons maintenant considérer une classe de catégories avec une $(\mathcal{T}, J)$-décomposition particulière, à savoir la décomposition normale, cf. [13], $\mathfrak{J}$ étant la classe de tous les épimorphismes normaux au sens de 
([8], § 8) et $J$, la classe de tous les monomorphismes. Dorénavant nous désignerons par $\mathcal{N}$ cette classe d'épimorphismes normaux, et nous considérerons les sous-catégories $\boldsymbol{N}$-réflexives, $\boldsymbol{N}$ étant la sous-catégorie coextensive dont les morphismes sont tous les épimorphismes normaux.

§ 1. Axiomatique. Dans ce paragraphe, nous reprenons avec quelques modifications les axiomes et plusieurs propriétés qui en découlent de l'article de Šul'geifer [13].

Soit une catégorie $\boldsymbol{C}$. Pour nous, un sous-objet $[K, u]$ d'un objet $A$ de $C$ est la classe de tous les couples $\left(K^{\prime}, u^{\prime}\right), u^{\prime}: K^{\prime} \rightarrow A$ étant un monomorphisme, équivalents à $(K, u)$. Un sous-objet $[K, u]$ sera noté souvent par $K$, en sous-entendant le monomorphisme $u$. L'image d'un sousobjet $[K, u]$ de $A \in \mathrm{Ob} C$ par $f: A \rightarrow B \in C$ est le sous-objet, s'il existe, $[f(K), v]$ de $B$ tel que $v f^{\prime}: A \rightarrow f(K) \rightarrow B$ soit à égal $f u$, où $f^{\prime} \in \mathcal{N}$, cf. ([8], $\S 10)$. Un idéal d'un objet $A$ de $\boldsymbol{C}$ est un sous-objet de $A$ qui est le noyau d'un morphisme ayant $A$ comme domaine.

Nous supposons maintenant que dans $\boldsymbol{C}$ sont valides les axiomes suivants:

B1. Il existe un objet nul, noté 0 .

B2. Tout morphisme possède un noyau.

B3. Tout morphisme $f: A \rightarrow B$ possède une image, i.e. $f(A)$ existe.

B4. L'image d'un idéal par un morphisme de $\mathcal{N}$ est un idéal.

B5. La classe des idéaux de tout objet est un ensemble.

B6. Il existe un produit pour toute famille d'objets.

Dans une catégorie $\boldsymbol{C}$ avec l'axiomatique (B), on obtient un certain nombre de propriétés que nous utiliserons par la suite: la plupart sont démontrées par Sul'geifer [13] et les autres sont obtenues facilement des premières:

P1. Un morphisme de $\mathcal{N}$ est le conoyau de son noyau.

P2. Si uf est tel que u est dans $J$, alors le noyau de uf est égal à celui de $f$.

P3. Pour qu'un morphisme soit dans J, il faut et il suffit que son noyau soit 0 .

P4. Si $g f \in \mathcal{N}$ et $f$ un épimorphisme, alors $g \in \mathcal{N}$.

P5. Si $f$ est dans $\mathcal{N} \cap J$, alors $f$ est un isomorphisme.

P6. Soit $B$ un idéal de $A$; si $C$ est un sous-objet de $A$ tel que $B \leqslant C$, $B$ est aussi un idéal de $C$.

P7. Il y a une bijection entre les idéaux de $A \in \mathrm{Ob} C$ et les classes d'équivalence de morphismes de $\mathcal{N}$ ayant $A$ comme domaine $(f \sim g$, s'il existe un isomorphisme $e$ tel que $e f=g$ ).
Si $K$ est un idéal de $A \in \mathrm{Ob} C$, à $K$ correspond un $f: A \rightarrow B \in \mathcal{N}$ dont le noyau est $K$. Alors $f$ est noté $f: A \rightarrow A / K$; et $A / K$ désigne le codomaine du morphisme $f: A \rightarrow A / K \in \mathcal{N}$ de noyau $K$.

P8. Le produit de deux morphismes de $\mathcal{N}$ est dans $\mathcal{N}$.

Soient $f: A \rightarrow A / K \in \mathcal{N}$ et $L$ un idéal de $A / K$. Si $g: A / K \rightarrow A / K / L$, alors l'image réciproque de $L$ par $f$, notée $f^{-1}(L)$, est par définition le noyau de $g f$.

P9. $f^{-1}$ établit une bijection entre les idéaux de $A / K$ et ceux de $A$ qui contiennent $K$; cette bijection préserve la relation d'ordre. Alors $f\left(f^{-1}(L)\right)=L$.

On note alors l'idéal $L$ de $A / K$ par $K^{\prime} \mid K$ où $K^{\prime}=f^{-1}(L)$ l'image réciproque de $L$.

P10. Soient $f: A \rightarrow A / K \in \mathcal{N}$ et $g: A \rightarrow A / L \in \mathcal{N}$; pour que $K \leqslant L$, il faut et il suffit qu'il existe $h: A / K \rightarrow A \mid L \in \mathcal{N}$ dont le noyau est $L / K$, i.e. $A / K / L / K=A \mid L$.

P11. Soit une famille de morphismes $\left\{f_{i}: A \rightarrow A / K_{i}\right\}$ de $\mathcal{N}$; alors le noyau de $f: A \rightarrow \prod_{I} A / K_{i}$ tel que $f_{i}=p_{i} f$ pour tout indice: $\left(p_{i}: \prod_{I} A / K_{i} \rightarrow\right.$ $\rightarrow A / K_{i}$, étant la $i^{e}$ projection) est $\bigwedge_{I} K_{i}$, le plus grand idéal de $A$ plus petit que tout $K_{i}$. Donc pour tout objet $A$ de $C$, l'ensemble des idéaux de $A$ forme un treillis complet.

P12. Si $f: A \rightarrow A / K$ est dans $\mathcal{N}$ et $L$ un idéal quelconque de $A$, alors $f(L)=L \vee K / K$.

De l'axiome B3 et des propriétés P4, P5 et P8 découle le corollaire suivant:

CoRollatre 2.1. Soit $\boldsymbol{C}$ une catégorie qui vérifie les axiomes B1 à B6: alors $\boldsymbol{C}$ est une catégorie avec une $(\mathcal{N}, \mathfrak{J})$-décomposition au sens de $\mathrm{I}, \S 2$.

§ 2. Radical dans une catégorie avec l'axiomatique (B). Dans ce paragraphe, on suppose que les axiomes B1 à B6 sont valides dans une catégorie $C$. On considère dans ce cas une sous-catégorie $\boldsymbol{B} \boldsymbol{N}$-réflexive quelconque de $\boldsymbol{C}$. Pour tout objet $A$ de $\boldsymbol{C}$ on définit le $\boldsymbol{B}$-radical de $A$, noté $r_{B}(A)$ ou simplement $r(A)$, comme étant le noyau de $\varrho_{A}: A \rightarrow R(A)$, la réflexion de $A$ dans $\boldsymbol{B}$.

Ainsi défini, le radical d'un objet de $\boldsymbol{C}$ peut être caractérisé de la façon suivante:

THÉoRÈme 2.2. Soit $r$ une fonction qui à tout $A \in \mathrm{Ob} C$ associe $r(A)$ un idéal de $A$. Alors pour que $r(A)$ soit le $\boldsymbol{B}$-radical de $A$ pour une souscatégorie $\boldsymbol{N}$-réflexive $\boldsymbol{B}$ de $\boldsymbol{C}$, il faut et il suffit que les conditions suivantes soient vérifiées:

(1) Pour tout $f: A \rightarrow B \in \mathcal{N}, f(r(A)) \leqslant r(B)$. 
(2) $r(a \mid r(A))=0$ pour tout $A \in \mathrm{Ob} \boldsymbol{C}$.

Démonstration. Supposons d'abord que $\boldsymbol{B}$ soit une sous-catégorie $N$-réflexive de $C$ et $r(A)=r_{B}(A)$, le noyau de $\varrho_{A}: A \rightarrow R(A) \in \mathcal{N}$, la réflexion de tout objet $A$ de $\boldsymbol{C}$ dans $\boldsymbol{B}$. Soit $f: A \rightarrow B \in \mathcal{N}$. Comme $\varrho: 1_{N} \rightarrow R$ est une transformation naturelle, on a $R(f) \varrho_{A}=\varrho_{B} f$. D'où $r(A) \leqslant f^{-1}(r(B))$ (P10). Donc $f(r(A)) \leqslant r(B)(\mathrm{P} 9)$. De plus $r(A / r(A))=r_{\boldsymbol{B}}(R(A))=0$ (P3) puisque $\varrho_{R(A)}$ est un isomorphisme. Réciproquement, soit $\boldsymbol{B}$, la souscatégorie pleine de $\boldsymbol{N}$ dont $\mathrm{Ob} \boldsymbol{B}=\{A \in \mathrm{Ob} \boldsymbol{C} \mid r(A)=0\}$. Alors $\boldsymbol{B}$ est une sous-catégorie $N$-réflexive de $C$. En effet, pour tout $A \in \mathrm{Ob} C$, posons $\varrho_{A}: A \rightarrow A / r(A)$ le morphisme de $\mathcal{N}$ correspondant à $r(A)$ et $R(A)$ $=A / r(A)$. Par construction $R(A) \in \mathrm{Ob} \boldsymbol{B}$ (condition (2)). De plus si $f$ : $A \rightarrow B \in \mathcal{N}$ tel que $B \in \mathrm{Ob} \boldsymbol{B}$, alors la condition (1) implique que $f(r(A))$ $\leqslant r(B)=0$ et, par conséquent en vertu de P12, $r(A) \leqslant \operatorname{ker} f$, le noyau de $f$; d'où il existe, par P10, $g: R(A) \rightarrow B$ tel que $g \varrho_{A}=f$. Et $g$ est unique puisque $\varrho_{A}$ est un épimorphisme. Donc $B \stackrel{R}{\stackrel{R}{\leftarrow}} N$.

Par cette caractérisation du radical d'un objet, nous retrouvons la définition d'un radical, utilisée par Michler [10] dans le cas de la théorie des anneaux. De la même manière, par extension, nous disons qu'une fonction $r$, qui à tout objet $A$ de $\boldsymbol{C}$ fait correspondre un idéal $r(A)$ de $A$, est un radical dans $C$ si les conditions (1) et (2) du théorème 2.2 sont satisfaites. Si $\boldsymbol{B}$ est une sous-catégorie $\boldsymbol{N}$-réflexive de $\boldsymbol{C}$ et $\boldsymbol{r}_{\boldsymbol{B}}$ le radical correspondant dans $\boldsymbol{C}$, la sous-catégorie pleine de $\boldsymbol{N}$ dont les objets ont leur radical nul est la sous-catégorie réflexive de $\boldsymbol{N}$ replète obtenue de $\boldsymbol{B}$ en ajoutant tous les objets isomorphes à ceux de $\boldsymbol{B}$ : c'est la sous-catégorie des objets $r_{\boldsymbol{B}}$-semi-simples, ou $r$-semi-simples.

Le théorème 1.3 fournit une construction d'un radical à partir d'une classe quelconque d'objets de $\boldsymbol{C}$ de la manière suivante:

THÉORÈme 2.3. Soit une classe $\mathfrak{B}$ d'objets de $C$. La fonction $r$ qui à tout $A \in \mathrm{Ob} C$ associe $r(A)=\bigcap\{K$, idéal de $A \mid$ il existe un isomorphisme e: $A / K \rightarrow B$ et $B \in \mathscr{B}\}$ est un radical dans $\boldsymbol{C}$, et les objets $r$-semi-simples sont des „sommes sous-directes" d'objets de $\mathfrak{B}$.

Démonstration. D'après l'axiome B5 et la propriété P7, $C$ est $\mathcal{N}$-„,cowell-powered" pour $\mathcal{B}$. D'après le théorème 1.3, la sous-catégorie $\boldsymbol{A}$ des $(\mathcal{N}, J)$-produits d'objets de $\mathcal{B}$ est une sous-catégorie $N$-réflexive de $C$. Alors pour tout $A \in \mathrm{ObC}, r_{A}(A)=\operatorname{Ker} \varrho_{A}=\operatorname{Ker} f, f: A \rightarrow \prod_{I} B_{i}$ tel que $p_{i} f=f_{i}: A \rightarrow B_{i} \in \mathcal{N}$ avec $B_{i} \in \mathcal{B}$ pour tout $i \in I$. Donc en vertu de P11, $r_{A}(A)=\bigcap\{K$, idéal de $A \mid$ il existe un isomorphisme $e: A / K \rightarrow B$ et $B \in \mathfrak{B}\}$. Par conséquent $r_{A}$ est un radical dont les objets $r_{A}$-semi-simples sont des $(\mathcal{N}, \mathfrak{J})$-produits d'objets de $\Re$, une généralisation évidente de la notion de sommes sous-directes, utilisée dans la théorie des anneaux.
Le corollaire du même théorème 1.3 établit le corollaire suivant:

CoRoliatre 2.4. Soit $\boldsymbol{B}$ une sous-catégorie $\boldsymbol{N}$-réflexive de $\boldsymbol{C}$. Si $\mathfrak{B}$ est une base de $\boldsymbol{B}$, alors pour tout $A \in \mathrm{Ob} C, r_{B}(A)=\bigcap\{K$, idéal de $A \mid$ il existe un isomorphisme e: $A / K \rightarrow B$ et $B \in \mathfrak{B}\}$.

Enfin la relation d'ordre sur la classe des sous-catégories $\boldsymbol{N}$-réflexives de $C$ induit une relation d'ordre sur les radicaux dans une catégorie.

Proposition 2.5. Soient $\boldsymbol{A}$ une sous-catégorie $\boldsymbol{N}$-réflexives de $\boldsymbol{C}$ et $\boldsymbol{B}$ une sous-catégorie $\boldsymbol{N}$-réflexive replète de $\boldsymbol{C}$. Pour que $\boldsymbol{A} \subseteq \boldsymbol{B}$, il faut et it suffit que $r_{B}(A) \leqslant r_{A}(A)$ pour tout $A \in \mathrm{Ob} \boldsymbol{C}$.

Démonstration. Supposons $\boldsymbol{A} \subseteq \boldsymbol{B}$ et soient $\varrho: 1_{N} \rightarrow R$ et $\sigma: 1_{N} \rightarrow S$, les réflexions de $\boldsymbol{N}$ dans $\boldsymbol{A}$ et dans $\boldsymbol{B}$ respectivement. Pour tout $A \in \mathrm{Ob} \boldsymbol{C}$, $\varrho_{A}$ est aussi dans $\boldsymbol{B}$, et par suite il existe $f: S(A) \rightarrow R(A) \in \mathcal{N}$ tel que $\varrho_{A}=f \sigma_{A}$. Donc d'après $\mathrm{P} 10, r_{B}(A) \leqslant r_{A}(A)$. Réciproquement si $r_{B}(A)$ $\leqslant r_{A}(A)$ pour tout $A \in \mathrm{Ob} C$, on a en particulier que pour tout $B \in \mathrm{Ob} \boldsymbol{A}$, $r_{\boldsymbol{B}}(B) \leqslant r_{\boldsymbol{A}}(A)=0$. Donc $B \in \mathrm{Ob} \boldsymbol{B}$, puisqu'il est $r_{\boldsymbol{B}}$-semi-simple et que $\boldsymbol{B}$ est replète.

§ 3. Dualité entre la classe des objets semi-simples et celles des objets radicaux. Dans ce paragraphe, nous considérons toujours une catégorie $C$ dans laquelle sont valides les axiomes B1 à B6. Soit $\boldsymbol{B}$ une sous-catégorie $\boldsymbol{N}$-réflexive de $\boldsymbol{C}$; on dit qu'un objet $\boldsymbol{A}$ de $\boldsymbol{C}$ est un objet $\boldsymbol{B}$-radical, ou simplement, un objet radical si $r_{B}(A)=A$, ou, ce qui revient au même, $R(A)=0$. On sait que la classe des objets $r_{B}$-semi-simples est celle des objets d'une sous-catégorie $\boldsymbol{N}$-réflexive de $\boldsymbol{C}$ et que le radical $r_{\boldsymbol{B}}$ en est ainsi caractérisé. Nous allons mäintenant étudier les conditions pour que la classe des objets radicaux soit la duale de la classe des objets semisimples, c'est-à-dire qu'elle soit la classe des objets d'une sous-catégorie $\boldsymbol{M}$-coréflexive de $\boldsymbol{C}, \boldsymbol{M}$ étant la sous-catégorie coextensive de $\boldsymbol{C}$ dont les morphismes sont les monomorphismes normaux au sens de ([8], \$ 8) de $C$.

A cette fin, nous supposons que dans $C$ est valide l'axiome suivant: B7. Le produit de deux monomorphismes normaux est un monomorphisme normal.

En vertu de l'axiome $\mathrm{B} 2$ et de $\mathrm{P} 6, \mathrm{~B} 7$ est équivalent à

B7'. Si $A$ est un idéal de $B$ et $B$ un idéal de $C$ pour des objets $A$, $B$ et $C$ de $C$, alors $A$ est un idéal de $C$.

On note que toute catégorie abélienne vérifie $B 7^{\prime}$.

Soit $\mathcal{M}$, la classe de tous les monomorphismes normaux de $\boldsymbol{C}$. Si $\boldsymbol{M}$ est la sous-catégorie coextensive de $\boldsymbol{C}$ constituée de tous les morphismes de $\mathcal{H}$, on définit par dualité qu'une sous-catégorie $\boldsymbol{A}$ de $\boldsymbol{C}$ est une souscatégorie $\boldsymbol{M}$-coréflexive de $\boldsymbol{C}$ si $\boldsymbol{A}$ est une sous-catégorie coréflexive de $\boldsymbol{M}$ avec $S: C \rightarrow M$ comme coréflecteur et $\sigma: S \rightarrow 1_{M}$ pour coréflexion. 
Si $\boldsymbol{B}$ est une sous-catégorie $\boldsymbol{N}$-réflexive de $\boldsymbol{C}$, soit $\boldsymbol{R}$, la sous-catégorie pleine de $\boldsymbol{M}$ dont les objets sont les objets $\boldsymbol{B}$-radicaux de $\boldsymbol{C}$; le théorème suivant découle immédiatement des définitions:

THÉ́onìme 2.6. Pour que la sous-catégorie $\boldsymbol{R}$ de $\boldsymbol{C}$ des objets $\boldsymbol{B}$-radicaux soit une sous-catégorie $\boldsymbol{M}$-coréflexive de $\boldsymbol{C}$, il faut et il suffit que les conditions suivantes soient vérifiées:

(1) $r_{B}(A)$ est un objet radical pour tout $A \in \mathrm{Ob} C$, i.e. $r_{B}^{2}=r_{B}$.

(2) pour tout $A \in \mathrm{Ob} C, r_{B}(A)$ est le plus grand idéal radical de $A$.

Nous appellerons idempotent un radical $r$ dans $\boldsymbol{C}$ qui vérifie la condition (1) du théorème précédent; complet un radical $r$ dans $\boldsymbol{C}$ qui vérifie la condition (2). Par conséquent pour un radical donné, la classe des objets semi-simple n'est la duale de celle des objets radicaux que si le radical est idempotent et complet dans une catégorie qui vérifie les axiomes B1 à B7. Nous utiliserons cependant les notions de radical idempotent et de radical complet, même si l'axiome B7 n'est pas vérifié dans $C$.

§4. Comparaison avec d'autres théories générales des radicaux. Nous comparerons notre théorie avec les autres dans le cas général d'une catégorie $\boldsymbol{C}$ qui vérifie les axiomes $\mathrm{B} 1$ à $\mathrm{B} 6$.

1. Les radicaux sous-foncteurs de l'identité. Soit $\boldsymbol{B}$ une sous-catégorie $\boldsymbol{N}$-réflexive replète de $\boldsymbol{C}$. D'après les propositions 1.5 et 1.6 pour que $\boldsymbol{B}$ soit une sous-catégorie réflexive de $\boldsymbol{C}$, il faut et il suffit que $\boldsymbol{B}$ contienne les sous-objets d'objets de $\boldsymbol{B}$ : ce qui donne, en traduisant en propriété du radical $r_{\boldsymbol{B}}=r$ correspondant à $\boldsymbol{B}$ :

Proposition 2.7. Soit $\boldsymbol{B}$ une sous-catégorie $\boldsymbol{N}$-réflexive replète àe $\boldsymbol{C}$. $\boldsymbol{B}$ est une sous-catégorie réflexive si et seulement si le radical correspondant $\boldsymbol{r}$ est un foncteur de $\boldsymbol{C}$ dans $\boldsymbol{C}$, sous-foncteur du foncteur identité.

Démonstration. Supposons que $r$ est un foncteur. Soit $B \in \mathrm{Ob} \boldsymbol{B}$; si $[A, u]$ est un sous-objet de $B$; soient $[r(A), v]$, et $[r(B), w]$, les radicaux de $A$ et de $B$. Alors $v u=w r(u)$ : ce qui implique que $r(A) \leqslant r(B)=0$. Done $A \in \mathrm{Ob} B$, puisque $\boldsymbol{B}$ est replète. Par conséquent, $\boldsymbol{B}$ est réflexive. Réciproquement supposons que $\boldsymbol{B}$ est une sous-catégorie réflexive de $C$ Soit $f: A \rightarrow B \in C$; on a alors le diagramme commutatif suivant:

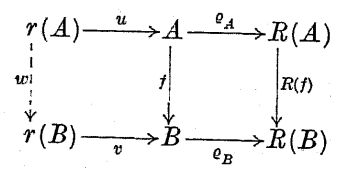

Puisque $[r(A), u]$ et $[r(B), v]$ sont respectivement les noyaux de $\varrho_{A}$ et de $\varrho_{B}, \varrho_{B} f u=R(f) \varrho_{A} u=0$. D'où il existe $w: r(A) \rightarrow r(B)$ tel que $f u=v w$, et $w$ est unique puisque $v$ est un monomorphisme. On peut donc poser $w=r(f)$. Il est facile de vérifier qu'on fait ainsi du radical un foncteur $r: \boldsymbol{C} \rightarrow \boldsymbol{C}$, sous-foncteur de l'identité de $\boldsymbol{C}$.

Les radicaux sous-foncteurs de l'identité sont les radicaux au sens de Dickson [4] et de Maranda [9]: ils correspondent donc aux sous-catégories réflexives replètes.

2. Les radicaux selon Amitsur. Une généralisation évidente d'un résultat de Maranda [9] donne le lemme suivant:

LEMIME 2.8. Si $r$ est un radical dans $C$, alors pour tout $A \in \mathrm{Ob} C$ et tout $A \rightarrow A / K \in \mathcal{N}$ tel que $K \leqslant r(A), r(A / K)=r(A) / K$

On considère maintenant une fonction $r$ qui à tout objet $A$ de $C$ associe un idéal $r(A)$ de $A$. Notons pour tout $A J(A)$, l'ensemble des idéaux $K$ de $A$ tels que $K \leqslant r(A)$.

Proposition 2.9. Pour que $r$ soit un radical dans $C$, il faut et il suffit que soient vérifiées les propriétés suivantes:

(1) Soit $f: A \rightarrow B \in \mathcal{N}:$ si $K \in \mathfrak{J}(A)$, alors $f(K) \in \mathfrak{J}(B)$.

(2) Pour tout $K \in J(A)$, il existe un idéal $L$ de $A$ tel que $L \leqslant K$ et tel que si $K^{\prime} \mid L \in \mathfrak{J}(A / L)$ et $K^{\prime} \leqslant K$, alors $K^{\prime}=L$.

Démonstration. Si $r$ est un radical, (1) est immédiate. Soit $K \notin J(A)$. Posons $L=r(A) \cap K$. Supposons que nous avons $M / L \in J(A / L)$, tel que $M / L \leqslant K / L$; alors $M / L \leqslant r(A / L)=r(A) / L$ (lemme 2.8). Donc $M \leqslant K$ et $M \leqslant r(A)$, c'est-à-dire $M \leqslant K \cap r(A)=L$. Donc $M / L=0$. Et la propriété (2) est vérifiée. Réciproquement si $r$ est une fonction telle que (1) et (2) sont vraies, évidemment $f(r(A)) \leqslant r(B)$ pour tout $f: A \rightarrow B \in \mathcal{N}$. De plus soit $K / r(A)=r(A / r(A))$. Pour tout idéal $L$ de $A$ tel que $L \leqslant K$, considérons $f: A \rightarrow A \mid L \in \mathcal{N}$. Si $r(A) \leqslant L$, alors d'après P12, l'image de $K / r(A)$ par le morphisme $A / r(A) \rightarrow A / L(\mathrm{P} 10)$ est $K / L$ dans $J(A / L)$ d'après (1). Si, par ailleurs, $r(A) \$ L$, alors $f(r(A))^{*}=r(A) \cup L / L(\mathrm{P} 12) \neq 0$ et $f(r(A)) \in J(A / L)$ et $f(r(A)) \leqslant K / L$. Donc, dans les deux cas, $K / L$ contient un idéal de $J(A / L)$. Par conséquent, d'après $(2), K \in J(A)$. Donc $r(A / r(A))=0$. Et $r$ est un radical dans $\boldsymbol{C}$.

CoRollaIRe 2.10. J est une RI-propriété selon Amitsur [1]. Réciproquement si $\mathfrak{J}$ est une RI-propriété selon Amitsur [1], la fonction $r$ qui à tout $A \in \mathrm{Ob} C$ associe $R_{A}$ le "radical" de $A$ est un radical.

En effet il suffit de constater que les résultats d'Amitsur sont valides dans la catégorie $\boldsymbol{C}$ et que l'objet nul 0 est évidemment dans les ensembles $\jmath(A)$ définis plus haut.

On remarque qu'à un même radical peuvent correspondre plusieurs RI-propriétés.

Fundamenta Mathematicae, T. LXXI 
3. Les propriétés radicales de Sul'geifer. Considérons une fonction $r$ qui à tout objet $A$ de $\boldsymbol{C}$ associe un idéal $r(A)$ de $A$. Un objet $A$ est dit $\mathcal{R}$-objet si $r(A)=A$.

Proposirion 2.11. Pour qu'une telle fonction $r$ soit un radical idempotent et complet (cf. $\S 3$ ), il faut et il suffit que

(1) l'image d'un $\mathfrak{R}$-objet par un morphisme soit un $\mathcal{R}$-objet.

(2) pour tout $A \in \mathrm{Ob} C, r(A)$ soit un $R$-idéal qui contient tous les $\Re$-idéaux de $A$, et $A / r(A)$ ne contienne pas d'autre $\mathcal{R}$-idéal que. 0 .

Démonstration. Si $r$ est un radical idempotent et complet, soient un $R$-objet $A$ et $f: A \rightarrow B \epsilon C$ : alors $f(r(A)) \leqslant r(f(A))$; or $r(A)=A$. D'où $r(f(A))=f(A)$, i.e. $f(A)$ est un $R$-objet. De plus si $K$ est un $R$-idéal de $A$, i.e. $r(K)=K$, alors $K \leqslant r(A), r$ étant complet. Puis si $K / r(A)$ est un $R$-idéal de $A / r(A), K / r(A) \leqslant r(A / r(A))=0$. Donc les conditions (1) et (2) sont nécessaires. Supposons en retour qu'elles soient vérifiées, alors $r(A)$ est un $\mathcal{R}$-objet pour tout $A \in \mathrm{Ob} C$; et de plus si $f: A \rightarrow B \in \mathcal{N}$, $f(r(A))$ est un $\mathcal{R}$-objet, ce qui implique que $f(r(A)) \leqslant r(B)$. Puisque $r(A / r(A))$ est un $R$-objet, alors $r(A / r(A))=0$. Donc $r$ est un radical et en vertu de la condition (2), il est idempotent et complet.

COROLLAIRE 2.12. La propriété $R$ ainsi définie vérifiant (1) et (2) est une propriété radicale au sens de Šul'geifer ([13], § 3). Réciproquement si $\mathcal{R}$ est une propriété radicale, la fonction $r$ qui à tout $A \in \mathrm{Ob} C$ associe $R_{A}$,le radical" au sens de Šul'geifer est un radical idempotent et complet.

Il suffit de constater qu'un objet $A$ de $C$ est un $\mathcal{R}$-objet si $R_{A}=A$. On note cependant que l'axiomatique de Šul'geifer [13] est plus faible que la nôtre.

En terminant ce paragraphe, nous pouvons remarquer qu'un radical sous-foncteur de l'identité dans une catégorie $\boldsymbol{C}$ où sont vérifiés les axiomes B1 à B6 est un radical complet. L'inverse n'est paś nécessairement vrai. Ainsi considérons le radical de Jacobson pour les anneaux (cf. [5]): c'est un radical idempotent et complet: pourtant il n'est pas un sous-foncteur de l'identité, car le sous-anneau des nombres rationnels de la forme $2 x /(2 y+1)$ où $x$ et $y$ sont des entiers est un anneau radical ([5], p. 103). D'autre part, on vérifie facilement cf. ([7], p. 102) que la fonction qui à tout groupe fait correspondre son sous-groupe commutateur est un radical sous-foncteur de l'identité: cependant c'est un radical non-idempotent. Enfin il existe des radicaux idempotents qui ne sont pas complets. Soit dans la catégorie de tous les anneaux la fonction $r$ ainsi définie: $r(A)=A$ si $A$ est un idéal principal de lui-même; $\sin o n r(A)=0$. Si $f: A \rightarrow B$ est un homomorphisme surjectif et que $A=(x)$, alors $B=(f(x))$. Donc $r$ est un radical idempotent. Par ailleurs, soit $P$ l'annean des entiers pairs: comme $m P \neq P$ pour tout $m \in P$, alors $r(P)=0$. Cependant $P$ contient des idéaux principaux non nuls. Donc $r$ n'est pas complet.

\section{Radicaux dans la catégorie des anneaux avec élément-unité}

Nous appliquons maintenant les résultats de la première section à une catégorie particulière, $\boldsymbol{U}$, de tous les anneaux avec élément-unité et des homomorphismes qui appliquent l'unité sur l'unité. Dans $U$ les axiomes $\mathrm{B} 2, \mathrm{~B} 3$ et $\mathrm{B} 4$ de $\mathrm{II} \S 1$, ne sont pas valides et, par suite, les théories générales des radicaux ne s'y appliquent pas.

§ 1. Radicaux d'anneaux avec élément-unité. Soient les classes $\delta$ des homomorphismes surjectifs de $\boldsymbol{U}$ et $\mathfrak{J}$ des homomorphismes injectifs de $\boldsymbol{U}$. Il est évident que $\boldsymbol{U}$ possède alors une $(\mathcal{S}, J)$-décomposition au sens de I, $\S 2$. Nous pouvons dans ce cas considérer les sous-catégories $\boldsymbol{S}$-réflexives de $\boldsymbol{U}, \boldsymbol{S}$ étant la sous-catégorie coextensive de $\boldsymbol{U}$ formée des morphismes de $\delta$.

Soit $\boldsymbol{B}$ une sous-catégorie $\boldsymbol{S}$-réflexive de $\boldsymbol{U}$, avec $\varrho: 1_{S} \rightarrow R$, la réflexion de $\boldsymbol{S}$ dans $\boldsymbol{B}$. Pour tout anneau $\boldsymbol{A}$ de $\boldsymbol{U}$, on appelle l'idéal bilatère Ker $\varrho_{A}$ le $\boldsymbol{B}$-radical de $A$, noté comme d'habitude $r_{\boldsymbol{B}}(A)$, ou simplement $r(A)$. On remarque que $r(A)$ n'est pas un objet de $\boldsymbol{U}$. Les anneaux dont le radical est nul sont appelés $r$-semi-simples et il est évident que tout $A \in \mathrm{Ob} \boldsymbol{B}$ est $\mathrm{r}$-semi-simple. C'est uniquement en ce sens que nous pouvons parler des radicaux dans la catégorie $\boldsymbol{U}$.

On sait que $U$ possède des produits quelconques, l'anneau $\{0,1\}$ étant l'objet nul de $\boldsymbol{U}$. Comme par ailleurs, pour tout anneau $A$ de $\boldsymbol{U}$, la classe des anneaux-quotients de $A$ est toujours un ensemble, il est évident que $\boldsymbol{U}$ est $\delta$ - „cowell-powered" pour toute classe d'anneaux de $\boldsymbol{U}$. Les conditions du théorème 1.3 sont satisfaites; et en le traduisant en termes de radical, nous obtenons que le théorème 2.3 est aussi valide pour la catégorie $\boldsymbol{U}$ :

THF́orìme 3.1. Soit une classe $\Re$ d'anneaux avec élément-unité. Pour tout anneau $A$ de $\boldsymbol{U}, r(A)=\bigcap\{I$, idéal bilatère de $A \mid$ il existe un isomorphisme $e: A \mid I \rightarrow B$ et $B \in \Re\}$ est le radical de $A$ dont les anneaux semisimples sont les "sommes sous-directes" d'anneaux de $\Re$.

En effet, selon la construction du théorème 1.3, il ne reste plus qu'à vérifier que l'homomorphisme canonique $A \rightarrow A / r(A)$ est bien la réflexion de cet anneau dans la sous-catégorie $S$-réflexive des $(\mathcal{S}, J)$-produits d'anneaux de $\mathfrak{B}$, c'est-à-dire des "sommes sous-directes".

On peut plonger $\boldsymbol{U}$ dans la catégorie $\boldsymbol{A}$ de tous les anneaux et de tous les homomorphismes. Cette dernière vérifie les axiomes B1 à B6 
de la section précédente, et la théorie générale des radicaux s'appliquent à $\boldsymbol{A}$. Nous allons maintenant à l'aide du théorème 1.8, établir une correspondance entre le radical d'un anneau de $\boldsymbol{U}$ défini par une sous-catégorie $\boldsymbol{S}$-réflexive de $\boldsymbol{U}$ et le radical de cet anneau défini par une souscatégorie $\mathcal{N}$-réflexive de $A, \mathcal{N}$ étant la classe des épimorphismes normaux de $\boldsymbol{A}$; ce qui revient à dire que $\mathcal{N}$ est la classe des homomorphismes surjectifs de $\boldsymbol{A}$. Pour cela nous avons besoin du lemme suivant:

Lemme 3.2. Soit $J$ le foncteur-inclusion de $U$ dans $A$, la catégorie de tous les anneaux. $J$ possède un adjoint à gauche $S$ tel que tout $f \in \mathcal{N}, S(f) \in \delta$.

Démonstration. Pour tout $A \in \mathrm{Ob} \boldsymbol{A}$, on pose $S(A)=A+Z$, l'ensemble des couples $(a, m), a \in A$ et $m \in Z$, l'anneau des entiers; $A+Z$, muni des opérations:

$$
\begin{aligned}
(a, m)+(b, n) & =(a+b, m+n), \\
(a, m)(b, n) & =(a b+m b+n a, m n)
\end{aligned}
$$

est un anneau arec élément-unité. Soit $\varkappa_{A}: A \rightarrow S(A)$ où $\varkappa_{A}(x)=(x, 0)$ pour tout $x \in A: A$ est ainsi identifié à un sous-anneau de $S(A)$. Si $f$ : $A \rightarrow B \in \boldsymbol{A}$ avec $B \in \mathrm{Ob} \boldsymbol{U}$, on définit $g: S(A) \rightarrow B$ par $g(a, m)=f(a)+m e$ où $e$ est l'élément-unité de $B$. Alors $g$ est l'unique homomorphisme de $\boldsymbol{U}$ tel que $g \varkappa_{A}=f$. Donc $S$ est l'adjoint à gauche de $J$. Si $f: A \rightarrow C \in \mathcal{N}$, il est clair que $S(f)((a, n))=(f(a), n)$ et $S(f)$ est surjectif.

Pour simplifier, nous noterons $S(A)$ par $\bar{A}$. Grâce au lemme 3.2, on vérifie facilement que le théorème 1.8 s'applique à $J: U \rightarrow A$ et aux sous-catégories $\boldsymbol{S}$-réflexives de $\boldsymbol{U}$ : ce qui donne immédiatement:

THÉORÈME 3.3. Soit $\boldsymbol{B}$ une sous-catégorie $\boldsymbol{S}$-réflesive de $\boldsymbol{U}$. $\boldsymbol{B}$ induit une sous-catégorie $\boldsymbol{N}$-réflexive $\boldsymbol{C}$ de $\boldsymbol{A}$ telle que $r_{C}(A)=r_{B}(\bar{A}) \cap A$.

En effet $r_{C}(A)$ est le noyau de l'homomorphisme composé $\varrho_{\bar{A}} \varkappa_{A}$ : $A \rightarrow \bar{A} \rightarrow \bar{A} / r_{B}(A)$.

Nous pouvons considérer le radical induit $r_{C}$ dans la catégorie $\boldsymbol{A}$ comme le prolongement de $r_{\boldsymbol{B}}$ dans la catégorie $\boldsymbol{U}$ : on voit sans peine que ce n'est pas un prolongement au sens strict. Cependant le théorème suivant contient des conditions pour que l'on ait un véritable prolongement, c'est-à-dire que $r_{C}(A)=r_{B}(A)$ pour tout $A \in \mathrm{Ob} U$.

THÉORÈnE 3.4. Soit $\boldsymbol{B}$ une sous-catégorie $\boldsymbol{S}$-réflexive de $\boldsymbol{U}$. si le radical correspondant à $\boldsymbol{B}$ possède les propriétés suivantes,

(1) $r_{B}(\bar{A}) \subseteq A$ pour tout $A \in \mathrm{Ob} \boldsymbol{A}$,

(2) $r_{B}(A) \subseteq r_{B} \overline{\left(r_{B}(A)\right)}$ pour tout $A \in \mathrm{Ob} \boldsymbol{U}$,

(3) si $K$ est un idéal de $A \in \mathrm{Ob} \boldsymbol{U}$ et que $K \subseteq r_{\boldsymbol{B}}(\bar{K})$, alors $K \subseteq r_{\boldsymbol{B}}(A)$, alors $r_{C}(A)=r_{B}(\bar{A})$ pour tout $A \in \mathrm{Ob} U$ et $r_{C}$ est un radical idempotent et complet dans $\boldsymbol{A}$ tel que $r_{\boldsymbol{C}}(A)=r_{\boldsymbol{C}}(\bar{A})$ pour tout $A \in \mathrm{Ob} \boldsymbol{A}$.
Démonstration. Comme d'après (1) et (2), on a $r_{B}(\bar{A}) \subseteq A$ et $r_{\boldsymbol{B}}(\bar{A}) \subseteq r_{\boldsymbol{B}} \overline{\left.r_{\boldsymbol{B}}(\bar{A})\right)}$, alors la propriété (3) implique $r_{\boldsymbol{B}}(\bar{A}) \subseteq r_{\boldsymbol{B}}(A)$, pour tout $A \in \mathrm{Ob}$. Par ailleurs $r_{\boldsymbol{B}}(A)$ est aussi un idéal de $\bar{A}$ tel que d'après (2), $r_{B}(A) \subseteq r_{\boldsymbol{B}} \overline{\left(r_{B}(A)\right)}$ : ce qui implique d'après $(3), r_{B}(A) \subseteq r_{B}(\bar{A})$. Done $r_{B}(A)$ $=r_{B}(\overline{\bar{A}})$ pour tout anneau avec élément-unité. Par conséquent pour tout $A \in \mathrm{Ob} \boldsymbol{U}, r_{\boldsymbol{C}}(A)=r_{\boldsymbol{B}}(\bar{A}) \cap A=r_{\boldsymbol{B}}(\bar{A})=r_{\boldsymbol{B}}(A)$, et pour tout $A \in \mathrm{Ob} \boldsymbol{A}$, $r_{\boldsymbol{C}}(A)=r_{\boldsymbol{B}}(\bar{A})=r_{\boldsymbol{B}}(\overline{\bar{A}})=r_{\boldsymbol{C}}(\bar{A})$. De plus, si $A$ est un anneau quelconque, $r_{C}(A)=r_{B}(\bar{A}) \cap A=r_{B}(\bar{A})=r_{B} \overline{\left(r_{B}(\bar{A})\right)}=r_{C}\left(r_{C}(A)\right)$, donc $r_{C}$ est idempotent: Si $K$ est un idéal bilatère de $A \in \mathrm{Ob} \boldsymbol{A}$ tel que $r_{C}(K)=K$, alors $r_{B}(\bar{K})=K$ et la propriété $(3)$ implique que $K \subseteq r_{B}(\bar{A})=r_{C}(A)$. Donc $r$ est complet.

Les conditions (1), (2) et (3) sont nécessaires au sens suivant: si $\boldsymbol{C}$ est une sous-catégorie $\boldsymbol{N}$-réflexive de $\boldsymbol{A}$, il est évident que $\boldsymbol{B}=\boldsymbol{C} \cap \boldsymbol{U}$ est une sous-catégorie $\boldsymbol{S}$-réflexive de $\boldsymbol{U}$, alors

Proposition 3.5. Si le radical $r_{C}$, correspondant à une sous-catégorie $\boldsymbol{C} \boldsymbol{N}$-réflexive de la catégorie $\boldsymbol{A}$, est idempotent, complet et tel que $r_{C}(A)$ $=r_{C}(\bar{A})$ pour tout anneau de $\boldsymbol{A}$, alors $r_{\boldsymbol{B}}$, le radical défini par $\boldsymbol{B}=\boldsymbol{C} \cap \boldsymbol{U}$, est la restriction de $r_{c} \grave{a} \boldsymbol{U}$ et vérifie les conditions (1), (2) et (3) $d u$ théorème 3.4.

Démonstration. Par définition, $r_{B}(A)=r_{C}(A)$ pour tout $A \in \mathrm{Ob} \boldsymbol{U}$ : alors $r_{\boldsymbol{B}}(\bar{A})=r_{\boldsymbol{C}}(\bar{A})=r_{C}(A) \subseteq A$ pour $A \in \mathrm{Ob} \boldsymbol{A}$. Donc (1) est vérifiée. De plus pour tout $A \in \mathrm{Ob} U, \quad r_{B}(A)=r_{C}(A)=r_{C}\left(r_{C}(A)\right)=r_{C} \overline{\left(r_{C}(A)\right)}$ $=r_{\boldsymbol{B}} \overline{\left(r_{\boldsymbol{B}}(A)\right)}$, puisque $r_{C}$ est idempotent: d'où (2) est aussi vérifiée. Pour vérifier la condition (3), soit $K$ un idéal de $A \in \mathrm{Ob} \boldsymbol{U}$ tel que $K=r_{\boldsymbol{B}}(\bar{K})$; comme $r_{B}(\bar{K})=r_{C}(\bar{K})=r_{C}(K)=K$, alors $K \subseteq r_{C}(A)$, puisque $r_{C}$ est complet: d'où $K \subseteq r_{\boldsymbol{B}}(A)$.

La condition $r_{C}(A)=r_{C}(\bar{A})$ n'est pas trop restrictive puisque comme l'a constaté Michler [10], elle est vérifiée chaque fois que $r_{C}(Z)=0$ pour l'anneau $Z$ des entiers, ce qui est le cas de la plupart des radicaux idempotents et complets de la théorie des anneaux. Par conséquent, pour définir un radical idempotent, complet (propriété radicale) et nul pour l'anneau des entiers, il suffit de le faire pour les anneaux avec élément-unité. Nous appliquerons ce résultat au paragraphe suivant.

§ 2. Classes d'idéaux à droite qui définissent des radicaux d'anneau. Pour tout $A \in \mathrm{Ob} \boldsymbol{U}$, considérons un ensemble d'idéaux à droite $\mathrm{J}(A)$ de $A$. Posons $r(A)=A$ si $J(A)=\emptyset$, sinon $r(A)=\bigcap_{J(A)} K$.

Proposinion 3.6. La sous-catégorie $\boldsymbol{B}$ pleine de $\boldsymbol{S}$ dont $\mathrm{Ob} \boldsymbol{B}=\{A \in \mathrm{Ob} \boldsymbol{U} \mid$ $r(A)=0\}$ est une sous-catégorie $\boldsymbol{S}$-réflexive de $\boldsymbol{U}$, si les ensembles $J(A)$, $A \in \mathrm{Ob} \boldsymbol{U}$, vérifient les propriétés suivantes:

(1) $r(A)$ est un idéal bilatère pour tout $A \in \mathrm{Ob} U$. 
(2) si $f: A \rightarrow B \in \boldsymbol{S}$ et $B \in \mathrm{Ob} \boldsymbol{B}$, pour tout $L \in \mathcal{J}(B), f^{-1}(L) \in \mathcal{J}(A)$.

(3) si $K \in \mathcal{J}(A), K / r(A) \in \mathcal{J}(A / r(A))$ pour tout $A \in \mathrm{Ob} \boldsymbol{U}$.

De plus $r(A)=\bigcap_{\mathcal{J}(A)}(0: A / K)$, où $(0: A / K)=\{a \in A \mid A a \subseteq K\}$.

Démonstration. On constate d'abord que $A / r(A) \in \mathrm{Ob} \boldsymbol{B}$. En effet

$$
\begin{aligned}
r(A / r(A))=\bigcap_{\mathcal{J}(A / r(A))} K / r(A) & \subseteq \bigcap_{\mathcal{J}(A)}(K / r(A)) \quad \text { d'après (3) } \\
& \left.\subseteq \bigcap_{\mathcal{J}(A)} K\right) / r(A)=0 .
\end{aligned}
$$

De plus soit $f: A \rightarrow B \in S$ tel que $r(B)=0$. Il suffit de vérifier que $f(r(A))=0$. De fait, si $L$ est le noyau de $f$,

$$
\begin{aligned}
f(r(A))=r(A) \cup L / L & =\left(\bigcap_{J(A)} K\right) \cup L / L \\
& \subseteq\left(\bigcap_{J(B)} f^{-1}\left(K^{\prime}\right)\right) \cup L / L \quad \text { d'après }(2) \\
& \subseteq \bigcap_{J(B)}\left(f^{-1}\left(K^{\prime}\right) \cup L\right) / L=\bigcap_{\Im(B)} f^{-1}\left(K^{\prime}\right) / L \\
& =f^{-1}\left(\bigcap_{J(B)} K^{\prime}\right) / L
\end{aligned}
$$

or $f^{-1}\left(\bigcap_{\mathcal{S}(B)} K^{\prime}\right)=f^{-1}(0)=L$. Done $f(r(A))=0$. De plus si $x \in A$ tel que $A x \subseteq \mathcal{K}$, i.e. $x \in(0: A / K)$ pour tout $K \in \mathcal{J}(A)$, alors $x \in K$; d'où $\bigcap_{J(A)}(0: A / K)$ $\subseteq \bigcap_{J(A)} K=r(A)$. Réciproquement $r(A) \subseteq(0: A / r(A)), r(A)$ étant un idéal bilatère; d'où pour tout $x \in r(A), A x \subseteq \bigcap_{\mathfrak{J}(A)} K \subseteq K$ pour tout $K \in \mathfrak{J}(A)$. Done $r(A) \subseteq \bigcap_{\mathcal{J}(A)}(0: A / K)$ et, par conséquent, $r(A)=\bigcap_{\mathcal{J}(A)}(0: A / K)$.

Pour chaque $A \in \mathrm{Ob} \boldsymbol{U}$, soit $\boldsymbol{B}_{A}$ une sous-catégorie $N$-réflexive de la catégorie $\boldsymbol{M}_{A}$ des modules à droite unitaires sur $A$ : dans chaque catégorie $\boldsymbol{M}_{A}$, les axiomes $\mathrm{B} 1$ à $\mathrm{B} 6$ sont valides. De plus soit $\mathfrak{乃}_{A}$ une base de $\boldsymbol{B}_{A}$ pour tout anneau $A$ de $\boldsymbol{U}$. D'après le corollaire $2.4, r_{\boldsymbol{B}_{A}}(A)=\bigcap\{K$, idéal à droite de $A \mid$ il existe $B \in \Re_{A}$ isomorphe à $\left.A / K\right\}$. A l'aide de la proposition 3.6, nous pouvons déterminer les conditions sur les classes $\mathcal{B}_{A}$ pour que la sous-catégorie pleine $\boldsymbol{C}$ de $\boldsymbol{S}$ dont $\mathrm{Ob} \boldsymbol{C}=\left\{\boldsymbol{A} \in \mathrm{Ob} \boldsymbol{U} \mid r_{\boldsymbol{B}_{A}}(A)\right.$ $=0\}$ soit une sous-catégorie $\boldsymbol{S}$-réflexive de $\boldsymbol{U}$ :

Proposition 3.7. Pour tout $A \in \mathrm{ObU}$, soit $J(A)=\{K$ idéal à droite de $A \mid$ il existe $B \in \Re_{A}$ isomorphe $\left.\grave{a} A / K\right\}$; alor's les ensembles $J(A)$ vérifient les propriétés de 3.6 si les conditions suivantes sont valides:

(1) $\Re_{A}$ est héréditaire pour tout $A \in \mathrm{Ob} U$.

(2) Sif: $A \rightarrow B \in \boldsymbol{S}$ tel que $B \in \mathrm{Ob} \boldsymbol{C}$, alors $B_{\mid} K \in \Re_{B}$ implique $B / K \in \mathfrak{B}_{A}$.

(3) Pour tout idéal à droite $K$ de $A \in \mathrm{Ob} \boldsymbol{U}$ tel que $A / K \in \mathfrak{B}_{A}$, alors $A \mid K \in \Re_{\mathcal{A} /(A)}$.
Démonstration. D'abord $r_{B_{A}}(A)$ est un idéal bilatère de $A$ pour tout $A \in \mathrm{Ob} \boldsymbol{U}$. Puisque $\Re_{A}$ est héréditaire, $r=r_{B_{A}}$ est un radical sousfoncteur de l'identité (proposition 2.7). Dans $\boldsymbol{M}_{A}$, soient $\varrho_{A}: A \rightarrow A / r(A)$ la réflexion de $A$ dans $\boldsymbol{B}_{A}$ et pour tout $a \in A-r(A), g_{a}: A \rightarrow A / r(A)$ tel $g_{a}(b)=\bar{a} b$ pour tout $b \in A$. Alors pour tout $a \in A-r(A)$, il existe $h_{a}$ : $A / r(A) \rightarrow A / r(A)$ tel que $g_{a}=h_{a} \varrho_{A}$. D'où $g_{a}(r(A))=0$, i.e. $\operatorname{ar}(A) \subseteq r(A)$. Done $r(A)$ est aussi idéal à gauche. Pour vérifier la propriété (2) de 3.6, il suffit de remarquer que $K$ et $B$ peuvent considérés comme des $A$-modules et que $A / f^{-1}(K) \simeq B / K$ dans $\boldsymbol{M}_{A}$ : et pour la propriété (3), remarquons que $r(A) \cdot A / K=0$ pour tout $K \in J(A)$ : par suite, $A / K$ est aussi un $A / r(A)$-module qui est équivalent à $A / r(A) / K / r(A)$.

COROLLATRE 3.8. $r(A)=\bigcap_{\mathcal{B}_{A}}(0: M)$ pour tout anneau $A$ de $\boldsymbol{U}$ si les conditions précédentes sont vérifiées.

Démoṇstration. On sait que $r(A)=\bigcap_{\mathfrak{J}(A)}(0: A / K)$; donc $r(A)$ $\supseteq \bigcap_{B_{A}}(0: M)$. Soit $x \in \bigcap_{J(A)} K$. Soit un $A$-module queleonque $M$ de $\mathscr{B}_{A}$; pour tout $y \in M, A / B \approx y \stackrel{\mathcal{J}(A)}{\subseteq} M$, si $B=(0: y)=\{a \in A \mid y a=0\}$. Comme $y A$ est aussi dans $\Re_{A}$, alors $B \in J(A)$ et $x \in B$. Done $y x=0$ pour tout $y \in M$, i.e. $x \in(0: M)$ pour tout $M \in \mathfrak{B}_{A}$.

Corollatre 3.9. Pour tout $A \in \mathrm{ObU}$, soit $\Sigma_{A}$ une classe spéciale de A-modules unitaires, au sens d'Andrunakievič et de Rjabuhin [2]. Si $r$ est. le radical qu'elles définissent, alors $r(A)=\bigcap\{K$, idéal à droite de $A \mid$ il existe $B \in \Sigma_{A}$ isomorphe $\left.\grave{a} A / K\right\}$, à la condition qu'elles soient héréditaires.

Cela découle du corollaire 3.8 et du théorème 3.4 .

En conclusion, on remarque que le corollaire 3.8 est la généralisation des résultats de Bourbaki [3] concernant le radical de Jacobson et que les conditions de la proposition 3.7 ne concernent en somme qu'une certaine classe d'idéaux à droite des anneaux; elles sont donc plus générales que celles qui définissent les classes spéciales de modules selon Andrunakievič et Rjabuhin [2].

\section{Références}

[1] S. A. Amitsur, A general theory of radicals, II, Amer. J. Math. 76 (1954), p. 100-125.

[2] B. A. Andrunakievič, J. M. Rjabuhin, Special modules and special radicals, Soviet Math. Dokl. Akad. Nauk SSSR 147 (1962), p. 1790-1793.

[3] N. Bourbaki, Algèbre. Eléments de mathématiques, Chap. 8, Paris 1958.

[4] S. Dickson, A torsion theory for Abetian categories, Trans. Amer. Math. Soc. 121 (1966), p. 223-235.

[5] N. J. Divinsky, Rings and radicals, Toronto 1965.

[6] J. F. Kennison, Full reflective subcategories and generalized covering spaces, Illinois J. Math. 12 (1968), p. 353-365. 
[7] A. G. Kuroš, Theory of groups, Vol. 1, New York, 1956.

[8] A. G. Kurosch, A. Ch. Liwschitz, E. G. Schulgeifer, M. S. Zalenko, Zur Theorie der Kategorien, Berlin 1963.

[9] J. Marand a, Injective structures, Trans. Amer. Math. Soc. 110 (1964), p. 98-135.

[10] G. Michler, Radikale und Sockel, Math. Annalen 167 (1966), p. 1-48.

[11] B. Mitchell, Theory of categories, New York 1965.

[12] J. M. Rjabuhin, Radicaux dans les catégories (en russe), Mat. Issl. Akad. Nauk Moldav. SSR 2 (1967), p. 107-164

[13] E. G. Sul'geifer, La théorie générale des radicaux dans les catégories (en russe), Mat. Sb. 51 (93) (1960), p. 487-500.

UNIVERSITE DE MONTREAL

Reçu par la Rédaction le 21. 6. 1969.

\section{Metric spaces in which a strengthened form of Blumberg's theorem holds}

by

Jack B. Brown (Auburn, Ala.)

Introduction. Let the following statement be referred to as

Proposition A. If $f$ is a function from $X$ into $Y$, then there is a dense subset $D$ of $X$ such that $f \mid D$ is continuous.

Henry Blumberg first proved [2] that Proposition A holds if $X$ is the plane and $Y$ is the space $R$ of real numbers, and he later proved [3] that Proposition A holds if $X$ and $Y$ are Euclidean spaces. Blumberg observes in [3] that according to his construction, the set $D$ is countable, and that it cannot be made to be otherwise, for Sierpinski and Zygmund have shown ([9], p. 118; [10]) that if the continuum hypothesis is true, there exists a real function $f$ with domain $R$ such that if $D$ is any uncountable subset of $R$, then $f \mid D$ has a point of discontinuity. Block and Cargal [1] show that Proposition A holds for fairly general topological spaces $X$ and $Y$, with suitable restrictions on the categoric nature of the open sets in $X$. In [6] Goffman gave an example to show that $D$ cannot be constructed so as to make $f \mid D$ a homeomorphism from $D$ onto $f(D)$. He also gave an example [6] which shows that Proposition 4 of [5], which states that Proposition A holds for every pair of metric spaces $X$ and $Y$, is false. Bradford and Goffman [4] proved that if $X$ is a metric space, then Proposition A holds (where $Y$ is $R$ ) if and only if every open subset of $X$ is of second category.

The main purpose of this paper is to establish theorems analogous to those of Bradford and Goffman concerning the following two propositions, each of which is stronger than Proposition $\mathrm{A}$ (where $Y=R$ ).

Proposition B. If $f$ is a function from $X$ into $R$, then there exists an uncountably dense subset $W$ of $X$ and a dense subset $D$ of $W$ such that $f \mid W$ is continuous at each element of $D$.

Proposition C. If $f$ is a function from $X$ into $R$, then there exists a c-dense subset $W$ of $X$ and a dense subset $D$ of $W$ such that $f \mid W$ is continuous at each element of $D$. 